\title{
Intoxicação experimental por Metternichia princeps (Solanaceae) em coelhos ${ }^{1}$
}

\author{
Naiara B. Maran², Saulo A. Caldas ${ }^{3}$, Juliana S. Prado ${ }^{3}$, Aline D. Gomes ${ }^{3}$, \\ Carlos Hubinger Tokarnia ${ }^{4}$ e Marilene de Farias Brito ${ }^{5 *}$
}

\begin{abstract}
Maran N.B., Caldas S.A., Prado J.S., Gomes A.D., Tokarnia C.H. \& Brito M.F. 2012. [Experimental poisoning of rabbits by Metternichia princeps (Solanaceae).] Intoxicação experimental por Metternichia princeps (Solanaceae) em coelhos. Pesquisa Veterinária Brasileira 32(9):872-880. Setor de Anatomia Patológica, Instituto de Veterinária, Universidade Federal Rural do Rio de Janeiro, Seropédica, RJ 23890-000, Brazil. E-mail: marilene@ufrrj.br

Metternichia princeps, a tree that belongs to the Solanaceae family, occurs in the Atlantic Forest of Brazil, from the state of Rio de Janeiro to Bahia. In the county of Itaguaí, state of Rio de Janeiro, M. princeps was suspected to cause a lethal kidney disease in goats. The poisoning was experimentally reproduced in goats confirming the suspicion. Experiments were then performed in rabbits. Leaves of $M$. princeps were collected in the county of Itaguaí, where the poisoning in goats occurred. Eleven rabbits received the dried plant material, ten received the fresh plant and one rabbit served as control. The dehydrated leaves were ground and mixed with water. Initially the suspension was administered by stomach tube to nine rabbits and caused death of seven of them at doses from $0.125 \mathrm{~g} / \mathrm{kg}$ on. Two rabbits that got sick, but survived at doses of $0.0625 \mathrm{~g} / \mathrm{kg}$ and $0.125 \mathrm{~g} / \mathrm{kg}$, received doses of 0.5 and $0.25 \mathrm{~g} / \mathrm{kg}$ respectively, two months later and died, indicating that no tolerance to the plant poisoning effects developed. Additionally the dried leaves administered six months after collection to two rabbits at doses of 0.5 and $1 \mathrm{~g} / \mathrm{kg}$ respectively, caused death only of the rabbit that received the dose of $1 \mathrm{~g} / \mathrm{kg}$, indicating that the stored leaves lost toxicity. Fresh sprouts caused death in three of six rabbits at doses of $1.55 \mathrm{~g} / \mathrm{kg}$ to $3 \mathrm{~g} / \mathrm{kg}$. Fresh mature leaves caused death in one of four rabbits at the dose of $2 \mathrm{~g} / \mathrm{kg}$, what suggests that there is no difference in toxicity between mature leaves and sprouts. The dried leaves caused death of rabbits at smaller doses than fresh leaves, what can be explained by the way of administration of the plant; dried leaves were given within a few minutes, whilst fresh leaves were ingested during one or two days, a fact that may influence absorption of the toxic principle. In all experiments with the dried and fresh leaves (sprouts and mature leaves) of M. princeps, liver and heart lesions predominated. The course of the poisoning was peracute to acute. The rabbits became apathetic with pale and cyanotic mucosae and cold ears; they fell down with struggling movements, vocalization and spaced breathing and died. At postmortem examination, the liver was pale with marked hepatic lobules; the other organs were congested and blood vessels were ingurgitated. The histopathological examination revealed as the more important changes in the liver, intense, especially centrilobular congestion, severe swelling of the hepatocytes, and foci of incipient necrosis of hepatocytes. In the heart, beside congestion, small groups of eosiophilic cardiac fibers, with nuclear picnosis and vacuolation were found. In the kidney, congestion and swelling of epithelial cells
\end{abstract}

\footnotetext{
${ }^{1}$ Recebido em 10 de abril de 2012.

Aceito para publicação em 29 de maio de 2012.

Parte da Dissertação de Mestrado do primeiro autor

${ }^{2}$ Mestranda do Curso de Pós-Graduação em Medicina Veterinária, área de concentração Patologia Animal, Universidade Federal Rural do Rio de Janeiro (UFRRJ), BR 465 Km 7, Seropédica, RJ 23890-000, Brasil.
}

\footnotetext{
${ }^{3}$ Discentes do Curso de Pós-Graduação em Ciências Veterinárias, área de concentração em Sanidade Animal, UFRRJ, Seropédica, RJ.

${ }^{4}$ Departamento de Nutrição Animal e Pastagem, Instituto de Zootecnia, UFRRJ, Seropédica, RJ. E-mail: tokarnia@ufrrj.br

${ }^{5}$ Departamento de Epidemiologia e Saúde Pública, Instituto de Veterinária, UFRRJ, Seropédica, RJ. *Autor para correspondência: marilene@ufrrj.br
} 
was seen. The performed experiments show that rabbits are susceptible to poisoning with dried and fresh leaves of M. princeps. It is suggested that the toxic principle of M. princeps, responsible for the clinical and pathological picture in rabbits, may not be the same compound that causes poisoning in goats or that the rabbit reacts differently as react goats; rabbits showed liver and heart lesions whilst goats had kidney lesions.

INDEX TERMS: Poisonous plants, Metternichia princeps, Solanaceae, plant poisoning, rabbits, pathology.

RESUMO.- Metternichia princeps, árvore que pertence à família Solanaceae, ocorre na Mata Atlântica desde o estado do Rio de Janeiro até a Bahia. É conhecida popularmente com os nomes de "café-do-mato", "trombeteira" e "jasmin-do-morro". Em um estabelecimento no município de Itaguaí, RJ, suspeitou-se que $M$. princeps era responsável por doença renal letal em caprinos. A intoxicação foi reproduzida experimentalmente nessa espécie, confirmando a suspeita. Foram então realizados experimentos em coelhos. Folhas de M. princeps foram coletadas no município de Itaguaí/RJ, local onde foi diagnosticada a intoxicação em caprinos. Onze coelhos receberam a planta dessecada, dez receberam a planta fresca e um coelho serviu como controle. As folhas dessecadas de M. princeps foram trituradas e misturadas com água. Inicialmente, a suspensão foi administrada por via intragástrica através de sonda a nove coelhos e causou a morte de sete coelhos nas doses a partir de $0,125 \mathrm{~g} / \mathrm{kg}$. Dois coelhos que adoeceram mas sobreviveram às doses de 0,0625 e de $0,125 \mathrm{~g} / \mathrm{kg}$, dois meses mais tarde receberam doses de 0,5 e $0,25 \mathrm{~g} / \mathrm{kg}$, respectivamente, e morreram, o que indica que não houve desenvolvimento de tolerância à toxidez da planta. Adicionalmente, a planta dessecada, administrada a dois coelhos nas doses de 0,5 e $1 \mathrm{~g} / \mathrm{kg}$ seis meses após a coleta, causou a morte somente do coelho que recebeu a dose de $1 \mathrm{~g} / \mathrm{kg}$, indicando que a planta armazenada perde em toxidez. Os brotos frescos causaram a morte de três dos seis coelhos, nas doses de $1,55 \mathrm{~g} / \mathrm{kg}$ a $3 \mathrm{~g} / \mathrm{kg}$. As folhas maduras frescas causaram a morte de um dos quatro coelhos, na dose de $2 \mathrm{~g} / \mathrm{kg}$, o que sugere que não há diferença na toxidez entre a brotação e as folhas maduras. As folhas dessecadas causaram a morte dos coelhos em doses menores que as folhas frescas, o que talvez possa ser explicado pelo modo de administração da planta; as folhas dessecadas eram administradas em poucos minutos, enquanto que as folhas frescas eram ingeridas durante um a dois dias, o que influenciaria a rapidez da absorção do princípio tóxico da planta. Tanto nos experimentos com as folhas dessecadas quanto com as folhas frescas (brotos e folhas maduras), predominaram no quadro clínico-patológico alterações relativas ao coração e ao fígado. A evolução da intoxicação foi superaguda a aguda. Os coelhos tornaram-se apáticos, com mucosas pálidas a cianóticas e orelhas frias, até que subitamente se debatiam na gaiola, faziam movimentos de pedalagem, vocalizavam, apresentavam acentuada dispneia e morriam. À necropsia, o fígado apresentava-se com evidenciação da lobulação; os demais órgãos estavam congestos e os vasos sanguíneos ingurgitados. À histopatologia, as lesões mais relevantes foram: no fígado, intensa congestão, especialmente centrolobular, tumefação acentuada dos hepatócitos e focos de necrose incipiente de hepatócitos; no coração, além de congestão, fibras cardíacas com aumento de eosinofilia, com picnose nuclear, fibras cardíacas com vacuolização, e no rim, congestão e tumefação de células epiteliais. Esses experimentos demonstram que o coelho é sensível à intoxicação pelas folhas dessecadas e frescas de M. princeps. Sugere-se que o princípio tóxico responsável pelo quadro clínico-patológico da intoxicação por M. princeps em coelhos não seja o mesmo princípio responsável pela intoxicação nos caprinos, ou que os coelhos reagem de maneira diferente dos caprinos; nos coelhos predominam alterações cardíacas e hepáticas enquanto que em caprinos as lesões são renais.

TERMOS DE INDEXAÇÃO: Plantas tóxicas, Metternichia princeps, Solanaceae, intoxicação por plantas, coelho, patologia.

\section{INTRODUÇÃo}

No Brasil, as plantas nefrotóxicas de interesse pecuário mais importantes são Thiloa glaucocarpa da família Com-

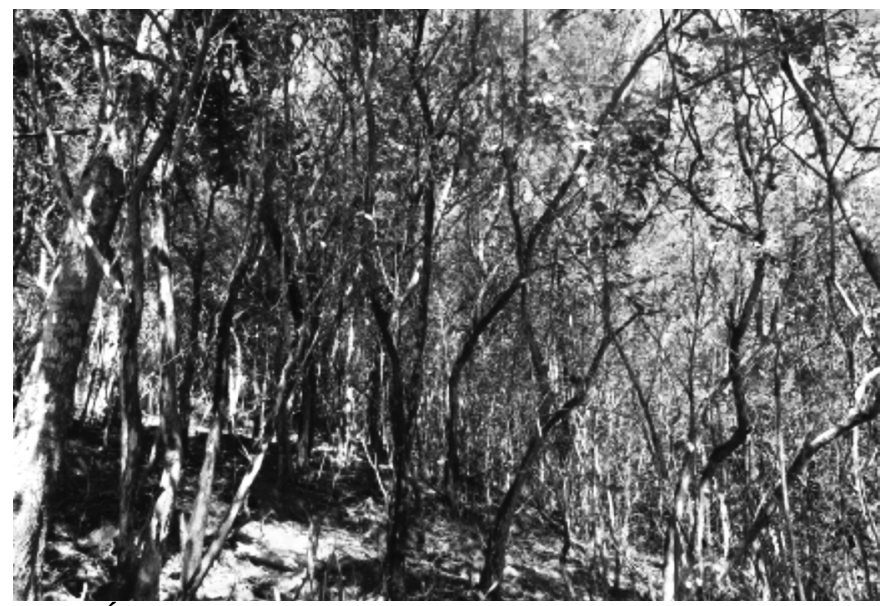

Fig.1. Área invadida por Metternichia princeps. Itaguaí, RJ.

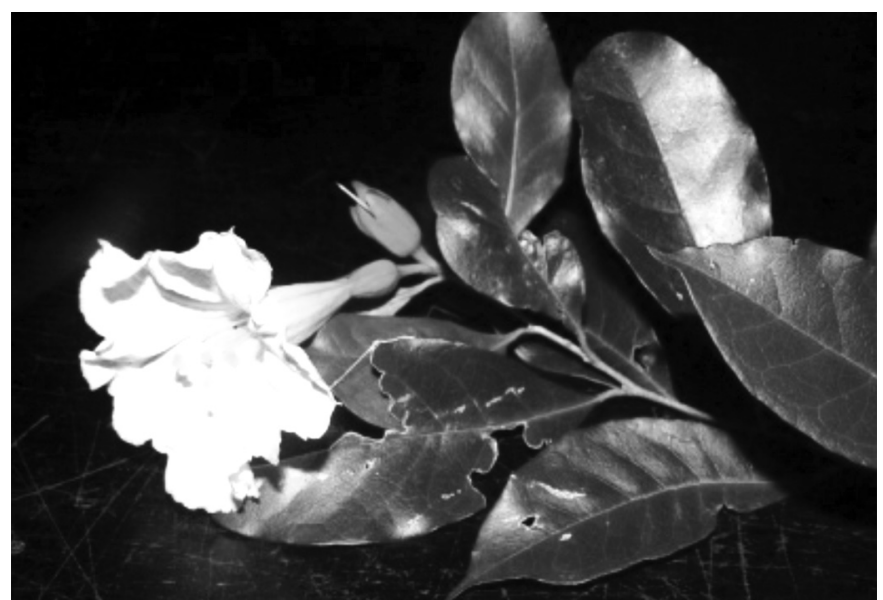

Fig.2. Inflorescência de Metternichia princeps. Itaguaí, RJ. 


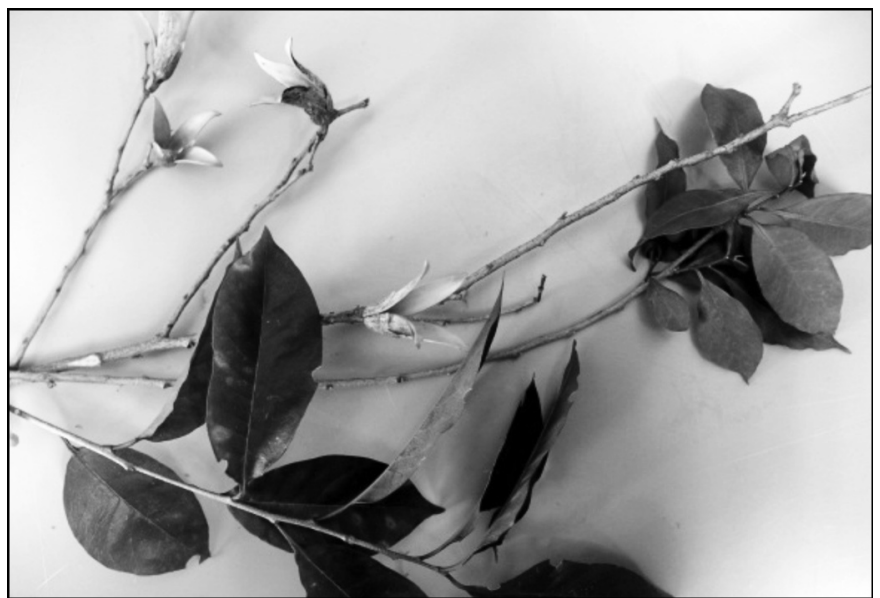

Fig.3. Metternichia princeps em frutificação. Itaguaí, RJ.

bretaceae, e diversas espécies do gênero Amaranthus, da família Amaranthaceae. Thiloa glaucocarpa ocorre nas caatingas, desde o Piauí e Ceará, até o oeste da Bahia e nordeste de Minas Gerais. Amaranthus spp ocorrem em todo Brasil. (Tokarnia et al. 2012).

No estado do Rio de Janeiro só havia sido registrado um surto causado por planta nefrotóxica, isto é, por Amaranthus spinosus, em ovinos (Peixoto et al. 2003).

Verificou-se a ocorrência de doença renal em caprinos causada por Metternichia princeps (Fig.1-3), uma Solanaceae, conhecida pelos nomes populares de "café-do-mato", "trombeteira" e "jasmin-do-morro" (Caldas et al. 2011, Caldas 2012, Prado 2012). Embora M. princeps esteja amplamente distribuída na Mata Atlântica, desde o estado do Rio de Janeiro até a Bahia, nunca fora associada a surtos de intoxicação em animais de fazenda até o ocorrido em caprinos no município de Itaguaí.

Este trabalho tem como objetivo determinar a sensibilidade do coelho a M. princeps, avaliar esta espécie como modelo experimental desta intoxicação e caracterizar o quadro clínico e anátomo-patológico.

\section{MATERIAL E MÉTODOS}

Para os experimentos com as folhas dessecadas foi realizada coleta no mês de abril de 2010 no município de Itaguaí/RJ, local onde foi diagnosticado o surto natural de intoxicação por Metternichia princeps em caprinos.

As folhas de $M$. princeps foram dessecadas à temperatura ambiente e à sombra por duas semanas. Depois de trituradas em liquidificador até a formação de pó, foram armazenadas em recipiente de vidro fechado, identificadas com a data de coleta e da moagem e mantidas sob refrigeração. A relação de peso entre as folhas maduras frescas: folhas dessecadas foi 3:1. Para experimentos com as folhas frescas foram feitas coletas nos meses de maio e junho de 2010.

0 processo de dessecação e trituração das folhas de M. princeps, bem como a experimentação, as necropsias e os exames histopatológicos foram realizados no Setor de Anatomia Patológica (SAP) do Projeto Sanidade Animal, Convênio Embrapa/UFRRJ, Seropédica, RJ.

Foram utilizados 22 coelhos adultos, da raça Nova Zelândia, com pesos variando entre 2,15 e 4,05 kg. Onze coelhos receberam a planta dessecada (Quadros 1-2), 10 receberam a planta fresca (Quadro 3) e um coelho serviu como controle (Quadro 2) (Protocolo da Comissão de Ética na Pesquisa da UFRRJ nº 094/2011).

Os coelhos foram mantidos em gaiolas individuais, alimentados com ração peletizada seca para coelhos ${ }^{6}$, capim e água à vontade. Nos experimentos em que a planta era administrada por via intragástrica, os coelhos foram mantidos em jejum prévio de um dia; nos experimentos com a planta fresca, o jejum prévio era de dois dias.

As folhas dessecadas, sob forma de pó, foram misturadas em $50 \mathrm{ml}$ de água e administradas por via intragástrica, conforme técnica descrita por Döbereiner et al. (1976). As folhas frescas inteiras (brotos e folhas maduras) foram oferecidas aos animais no dia da coleta e no dia seguinte após armazenamento em geladeira.

${ }^{6}$ Ração para coelho Natural Purina.

Quadro 1. Experimentos realizados em coelhos com folhas maduras dessecadas de Metternichia princeps

\begin{tabular}{|c|c|c|c|c|c|c|c|}
\hline № Coelho (Reg. SAP) & $\begin{array}{l}\text { Peso } \\
(\mathrm{kg})\end{array}$ & $\begin{array}{l}\text { Data e hora da } \\
\text { administração }\end{array}$ & $\begin{array}{c}\text { Quantidade (g) das } \\
\text { folhas maduras } \\
\text { dessecadas }\end{array}$ & $\begin{array}{l}\text { Dose } \\
\text { (g/kg) }\end{array}$ & $\begin{array}{l}\text { Início dos sinais } \\
\text { clínicos após } \\
\text { administração }\end{array}$ & Evolução & Desfecho \\
\hline 1449 (SAP 32065) & 2,90 & $\begin{array}{c}04 / 05 / 10 \\
09: 55\end{array}$ & 5,8 & 2 & $3 \mathrm{~h} \mathrm{e} 15 \mathrm{~min}$ & $14 \mathrm{~min}$ & morreu \\
\hline 1450 (SAP 32066) & 2,60 & $\begin{array}{c}04 / 05 / 10 \\
9: 44\end{array}$ & 5,2 & 2 & $4 \mathrm{~h}$ e $17 \mathrm{~min}$ & $5 \mathrm{~min}$ & morreu \\
\hline 1451 (SAP 32067) & 3,17 & $\begin{array}{c}05 / 05 / 10 \\
14: 25\end{array}$ & 3,17 & 1 & $2 \mathrm{~h} \mathrm{e} 45 \mathrm{~min}$ & $15 \mathrm{~min}$ & morreu \\
\hline 1452 (SAP 32068) & 3,25 & $\begin{array}{c}05 / 05 / 10 \\
14: 33\end{array}$ & 1,625 & 0,5 & $2 \mathrm{~h}$ & $1 \mathrm{~h}$ e $51 \mathrm{~min}$ & morreu \\
\hline 1453 (SAP 32069) & 3,25 & $\begin{array}{c}07 / 05 / 10 \\
11: 18\end{array}$ & 0,8125 & 0,25 & $7 \mathrm{~h}$ e $52 \mathrm{~min}$ & $1 \mathrm{~h}$ e $3 \min$ & morreu \\
\hline \multirow[t]{2}{*}{1454 (SAP 32121) } & 3,9 & $\begin{array}{c}07 / 05 / 10 \\
11: 22\end{array}$ & 0,4875 & 0,125 & $7 \mathrm{~h}$ e $2 \mathrm{~min}$ & $7 \mathrm{~h}$ e $27 \mathrm{~min}$ & recuperou-se \\
\hline & 4,05 & $\begin{array}{c}14 / 07 / 10 \\
15: 12\end{array}$ & 2,025 & 0,5 & $3 \mathrm{~h}$ & $24 \mathrm{~min}$ & morreu \\
\hline \multirow[t]{2}{*}{1455 (SAP 32122) } & 3,29 & $\begin{array}{c}11 / 05 / 10 \\
11: 24\end{array}$ & 0,205 & 0,0625 & $11 \mathrm{~h}$ e $38 \mathrm{~min}$ & $6 \mathrm{~h}$ e $32 \mathrm{~min}$ & recuperou-se \\
\hline & 3,39 & $\begin{array}{c}14 / 07 / 10 \\
14: 58\end{array}$ & 0,8475 & 0,25 & $3 \mathrm{~h}$ e $28 \mathrm{~min}$ & $42 \mathrm{~min}$ & morreu \\
\hline 1456 (SAP 32071) & 2,89 & $\begin{array}{c}11 / 05 / 10 \\
11: 17\end{array}$ & 0,7225 & 0,25 & $2 \mathrm{~h}$ e $33 \mathrm{~min}$ & $27 \mathrm{~min}$ & morreu \\
\hline 1457 (SAP 32072) & 3,08 & $\begin{array}{c}11 / 05 / 10 \\
11: 30\end{array}$ & 0,385 & 0,125 & $8 \mathrm{~h}$ e $7 \mathrm{~min}$ & $2 \mathrm{~h}$ e $24 \mathrm{~min}$ & morreu \\
\hline
\end{tabular}


Quadro 2. Experimentos realizados em coelhos com as folhas maduras dessecadas de Metternichia princeps e armazenadas por seis meses

\begin{tabular}{lccccccc}
\hline № Coelho (Reg. SAP) & $\begin{array}{c}\text { Peso } \\
\text { (kg) }\end{array}$ & $\begin{array}{c}\text { Data e hora da } \\
\text { administração }\end{array}$ & $\begin{array}{c}\text { Quantidade (g) das } \\
\text { folhas maduras } \\
\text { dessecadas }\end{array}$ & $\begin{array}{c}\text { Dose } \\
\text { (g/kg) }\end{array}$ & $\begin{array}{c}\text { Início dos sinais } \\
\text { clínicos após } \\
\text { administração }\end{array}$ & Evolução & Desfecho \\
\hline 1468 (SAP 32173) & 2,80 & $\begin{array}{c}21 / 10 / / 10 \\
09: 42\end{array}$ & 2,8 & 1 & $4 \mathrm{~h}$ & 2h e 43min & Morreu \\
$1469(-)$ & 2,72 & $\begin{array}{c}21 / 10 / 10 \\
9: 52\end{array}$ & 1,4 & 0,5 & $3 \mathrm{~h}$ e 50 min & - & Recuperou-se \\
1470 (SAP 32174) & 2,79 & $\begin{array}{c}21 / 10 / 10 \\
14: 2\end{array}$ & - & - & - & - & Eutanasiado \\
Controle & & & & & &
\end{tabular}

Quadro 3. Experimentos realizados em coelhos com brotos e folhas maduras de Metternichia princeps

\begin{tabular}{|c|c|c|c|c|c|c|c|c|c|}
\hline $\begin{array}{l}\text { № Coelho } \\
\text { (Reg. SAP) }\end{array}$ & $\begin{array}{l}\text { Peso } \\
(\mathrm{kg})\end{array}$ & $\begin{array}{l}\text { Estado de matu- } \\
\text { ração da planta }\end{array}$ & $\begin{array}{l}\text { Data e hora da } \\
\text { administração }\end{array}$ & $\begin{array}{l}\text { Quantidade total } \\
\text { da planta }(\mathrm{g})\end{array}$ & $\begin{array}{r}\text { Dose total in } \\
(\mathrm{g} / \mathrm{kg}\end{array}$ & ngerida & $\begin{array}{l}\text { Início dos sinais } \\
\text { clínicos após } \\
\text { oferta da planta }\end{array}$ & Evolução & Desfecho \\
\hline $1458(-)$ & 2,28 & Broto & $\begin{array}{c}11 / 05 / 10 \\
15: 33\end{array}$ & 5,2 & 2,36 & & - & - & s.a \\
\hline $1459(-)$ & 2,21 & Broto & $\begin{array}{c}11 / 05 / 10 \\
15: 53\end{array}$ & 4,05 & 1,8 & & - & - & s.a \\
\hline $1460(-)$ & 2,3 & Broto & $\begin{array}{c}11 / 05 / 10 \\
15: 54\end{array}$ & 6,58 & 2,8 & & - & - & s.a \\
\hline 1461 (SAP 32073) & 2,35 & Broto & $\begin{array}{c}12 / 05 / 10 \\
11: 32\end{array}$ & 7,12 & 3,0 & & $7 \mathrm{~h} \mathrm{e} 49 \mathrm{~min}$ & $1 \mathrm{~h}$ & Morreu \\
\hline 1462 (SAP 32075) & 2,34 & Broto & $\begin{array}{c}12 / 05 / 10 \\
11: 35\end{array}$ & 3,64 & 1,55 & & $11 \mathrm{~h}$ e $22 \mathrm{~min}$ & $3 \mathrm{~h}$ e $5 \mathrm{~min}$ & Morreu \\
\hline 1463 (SAP 32074) & 2,36 & Broto & $\begin{array}{c}12 / 05 / 10 \\
11: 37\end{array}$ & 5,66 & 2,4 & & $7 \mathrm{~h}$ e $39 \mathrm{~min}$ & $1 \mathrm{~h}$ e $6 \mathrm{~min}$ & Morreu \\
\hline $1464(-)$ & 2,15 & $\begin{array}{l}\text { Folha Madura } \\
\text { Folha Madura }\end{array}$ & $\begin{array}{c}20 / 07 / 10 \\
16: 50 \\
21 / 07 / 10 \\
11: 30\end{array}$ & $\begin{array}{l}2,15 \\
1,69\end{array}$ & $\left.\begin{array}{c}1,0 \\
0,78\end{array}\right\}$ & $=1,78$ & - & - & s.a \\
\hline 1465 (SAP 32124) & 2,3 & $\begin{array}{l}\text { Folha Madura } \\
\text { Folha Madura }\end{array}$ & $\begin{array}{c}20 / 07 / 10 \\
16: 50 \\
21 / 07 / 10 \\
11: 30\end{array}$ & $\begin{array}{l}2,3 \\
2,3\end{array}$ & $\left.\begin{array}{l}1,0 \\
1,0\end{array}\right\}$ & $=2,0$ & $11 \mathrm{~h}$ e $30 \mathrm{~min}$ & $2 \mathrm{~h}$ e $20 \mathrm{~min}$ & Morreu \\
\hline $1466(-)$ & 2,6 & $\begin{array}{l}\text { Folha Madura } \\
\text { Folha Madura }\end{array}$ & $\begin{array}{c}20 / 07 / 10 \\
16: 50 \\
21 / 07 / 10 \\
11: 30\end{array}$ & $\begin{array}{l}1,26 \\
1,99\end{array}$ & $\left.\begin{array}{l}0,48 \\
0,76\end{array}\right\}$ & $=1,24$ & - & - & s.a \\
\hline $1467(-)$ & 2,86 & $\begin{array}{l}\text { Folha Madura } \\
\text { Folha Madura }\end{array}$ & $\begin{array}{c}20 / 07 / 10 \\
16: 50 \\
21 / 07 / 10 \\
11: 30\end{array}$ & $\begin{array}{r}2,6 \\
0,93\end{array}$ & $\left.\begin{array}{l}0,90 \\
0,32\end{array}\right\}$ & $=1,22$ & - & - & s.a \\
\hline
\end{tabular}

Dos onze coelhos que receberam a planta dessecada por via intragástrica, nove a receberam após a coleta e dessecagem em doses que variaram de $0,125 \mathrm{~g} / \mathrm{kg}$ a $2 \mathrm{~g} / \mathrm{kg}$ (Quadro 1 ). Dois outros coelhos receberam as folhas dessecadas, trituradas e armazenadas por seis meses em vidro fechado, mantido sob refrigeração, nas doses de $1 \mathrm{~g} / \mathrm{kg}$ e 0,5g/kg (Quadro 2).

Dois coelhos, que adoeceram, mas sobreviveram às doses de $0,125 \mathrm{~g} / \mathrm{kg}$ e $0,0625 \mathrm{~g} / \mathrm{kg}$ (Coelhos 1454 e 1455) da planta dessecada, dois meses mais tarde receberam doses letais (respectivamente de $0,5 \mathrm{~g} / \mathrm{kg}$ e $0,25 \mathrm{~g} / \mathrm{kg}$ ), para verificar o eventual desenvolvimento de tolerância. (Quadro 1)

Dos dez coelhos que receberam as folhas frescas, seis coelhos ingeriram brotos (Coelhos 1458, 1459, 1460, 1461, 1462 e 1463), nas doses de $1,55 \mathrm{~g} / \mathrm{kg}$ a $3 \mathrm{~g} / \mathrm{kg}$, durante um período de um dia. Quatro coelhos (Coelhos 1464, 1465, 1466 e 1467) ingeriram as folhas maduras nas doses de $1,22 \mathrm{~g} / \mathrm{kg}$ a $2 \mathrm{~g} / \mathrm{kg}$, durante um período de dois dias. (Quadro 3).

Depois da administração da planta (dessecada por sonda, ou fresca em comedouro), os coelhos permaneceram em observação contínua por 48 horas. Os animais que apresentaram sinais clínicos e sobreviveram, depois desse período, não mais foram obser- vados continuamente, mas a intervalos de uma a duas horas até a total recuperação. Os animais que morreram, foram imediatamente necropsiados, e fragmentos de diversos órgãos foram coletados para exame histopatológico. Durante a necropsia, foi realizado, após a abertura da bexiga, exame de urina com a utilização de tiras reagentes para uroanálise ${ }^{7}$.

Os fragmentos coletados para histopatologia foram fixados em formol a $10 \%$ tamponado com carbonato de cálcio e processados pelos métodos usuais. Cortes com $5 \mu$ foram corados pela hematoxilina-eosina. Cortes do fígado foram submetidos à coloração pelo ácido periódico de Schiff (PAS).

\section{RESULTADOS}

Dos 21 coelhos que receberam folhas de Metternichia princeps, 14 morreram, três se recuperaram.

$\mathrm{Na}$ administração das folhas maduras dessecadas, doses únicas a partir de $0,25 \mathrm{~g} / \mathrm{kg}$ causaram a morte de todos os coelhos. A dose de 0,125g/kg causou a morte de um (Co-

\footnotetext{
${ }^{7}$ Multistix 10 SG, Bayer.
} 
elho 1457) de dois coelhos, o outro (Coelho 1454) adoeceu, mas se recuperou. A dose de 0,0625 causou sinais de intoxicação no único coelho (Coelho 1455) a que foi administrada a planta e que se recuperou. Os dois coelhos que adoeceram, mas se recuperaram (Coelhos 1454 e 1455), e que dois meses mais tarde receberam respectivamente doses letais de $0,5 \mathrm{~g} / \mathrm{kg}$ e $0,25 \mathrm{~g} / \mathrm{kg}$ para verificar uma eventual tolerância à toxidez da planta, morreram. (Quadro 1)

A planta dessecada administrada seis meses após a coleta causou a morte do coelho que recebeu a dose de $1,0 \mathrm{~g} / \mathrm{kg}$ (Coelho 1468), enquanto que o coelho que recebeu a dose de $0,5 \mathrm{~g} / \mathrm{kg}$ (Coelho 1469) adoeceu, mas se recuperou. (Quadro 2)

Dos seis coelhos que ingeriram brotos frescos da planta, três morreram; tinham ingerido doses de $3 \mathrm{~g} / \mathrm{kg}, 1,55 \mathrm{~g} / \mathrm{kg}$ e 2,4g/kg (Coelhos 1461, 1462 e 1463) (Quadro 3).

Dos quatro coelhos que ingeriram as folhas maduras frescas no período de dois dias, somente o que ingeriu $2 \mathrm{~g} / \mathrm{kg}$, adoeceu e morreu (Coelho 1465). Os outros três, que ingeriram $1,78 \mathrm{~g} / \mathrm{kg}, 1,24 \mathrm{~g} / \mathrm{kg}$ e $1,22 \mathrm{~g} / \mathrm{kg}$, não mostraram sinais de intoxicação (Coelhos 1464, 1466 e 1467) (Quadro 3).

Em todos os experimentos realizados a sintomatologia observada foi bastante uniforme, independente da forma de administração da planta (folhas frescas ou dessecadas).

Os coelhos que adoeceram e morreram (Quadros 1-3) apresentaram inicialmente letargia e estavam apáticos na gaiola, sem interação com o ambiente, ficavam deitados sem manifestar reações ao serem estimulados (toque, barulho). Aos poucos, adotavam posturas anormais, com a cabeça apoiada no solo, orelhas caídas e as pernas abertas. Notava-se palidez de mucosas e cianose das orelhas e do focinho, congestão severa de vasos episclerais e das orelhas, que se apresentavam frias, até que, subitamente, iniciava-se um quadro convulsivo intenso; os coelhos ficavam em decúbito lateral e faziam fortes movimentos de pedalagem com todos os membros (Fig.4.) e tinham tremores musculares generalizados. Apresentavam acentuado opistótono, esticavam todo o corpo e os membros, apresentavam fortes contrações abdominais até girarem sobre o eixo do próprio corpo e vocalizavam em tom muito alto. Esse quadro era alternado com momentos de dispneia intensa e respiração predominantemente abdominal e os coelhos abriam a boca em crises de apneia. Por fim, apresentavam novamente movimentos de pedalagem, opistótono, contrações musculares seguidas de relaxamento e morriam.

Os Coelhos 1454 e 1455 (Quadro 1) que receberam as folhas maduras dessecadas e que se recuperaram em até 12 horas, apresentaram sinais clínicos semelhantes aos coelhos que morreram.

O Coelho 1469 (Quadro 2) que recebeu as folhas maduras dessecadas e armazenadas por seis meses e que se recuperou em até 6 horas, também apresentou os mesmos sintomas.

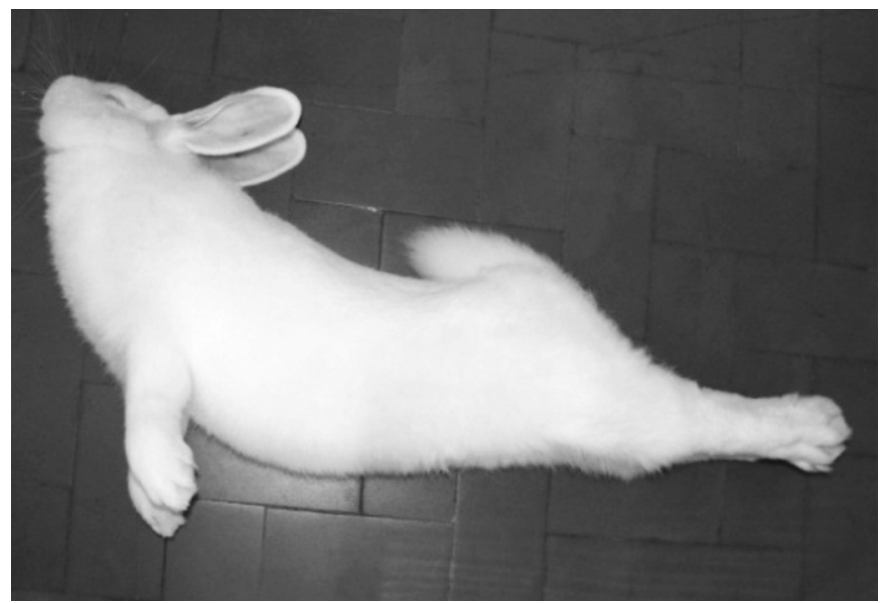

Fig.4. Movimentos de pedalagem na intoxicação experimental $(2 \mathrm{~g} / \mathrm{kg})$ por Metternichia princeps. Coelho 1449 (SAP 32065).

Quadro 4. Análise de urina na intoxicação experimental por Metternichia princeps em coelhos

\begin{tabular}{|c|c|c|c|c|c|c|c|c|c|c|}
\hline & Glicose & Bilirrubina & $\begin{array}{c}\text { Corpos } \\
\text { cetônicos }\end{array}$ & Densidade & Sangue & $\mathrm{pH}$ & Proteína & Urubilinogênio & Nitrito & Leucócitos \\
\hline № Coelho (Reg. SAP) & \multicolumn{10}{|c|}{ Folhas dessecadas } \\
\hline 1449 (SAP 32065) & $-{ }^{a}$ & - & - & 1030 & - & 6,5 & ++ & - & - & - \\
\hline 1450 (SAP 32066) & - & - & - & 1015 & - & 6,5 & ++ & - & - & - \\
\hline 1451 (SAP 32067) & - & + & - & 1015 & - & 7,0 & ++ & - & - & - \\
\hline 1452 (SAP 32068) & - & $(+)$ & - & 1030 & - & 6,0 & ++ & - & - & - \\
\hline 1453 (SAP 32069) & - & - & $(+)$ & 1030 & - & 6,5 & ++ & - & - & - \\
\hline 1454 (SAP 32121) & - & - & - & 1015 & - & 6,5 & + & - & - & - \\
\hline 1455 (SAP 32122) & - & - & - & 1010 & - & 7,0 & ++ & - & - & - \\
\hline 1456 (SAP 32071) & - & $(+)$ & - & 1030 & - & 6,5 & + & - & - & - \\
\hline \multirow[t]{2}{*}{1457 (SAP 32072) } & - & $(+)$ & - & 1030 & - & 6,0 & ++ & - & - & - \\
\hline & \multicolumn{10}{|c|}{ Folhas maduras dessecadas e armazenadas por 6 meses } \\
\hline 1468 (SAP 32173) & - & + & - & 1030 & - & 6,0 & +++ & - & - & - \\
\hline 1470 (SAP 32174) & - & + & - & 1030 & - & 6,5 & + & - & - & - \\
\hline 1474 (SAP 32075) & - & - & 1030 & - & 6,5 & + & - & - & - & - \\
\hline & \multicolumn{10}{|c|}{ Brotos e folhas maduras frescas } \\
\hline 1461 (SAP 32073) & - & + & - & 1030 & - & 6,0 & +++ & - & - & - \\
\hline 1462 (SAP 32075) & - & + & - & 1030 & - & 6,0 & + & - & - & - \\
\hline 1463 (SAP 32074) & - & $(+)$ & - & 1030 & - & 6,5 & ++ & - & - & - \\
\hline 1465 (SAP 32124$)$ & - & $(+)$ & - & 1030 & - & 6,0 & ++ & - & - & - \\
\hline
\end{tabular}


A evolução desses sinais clínicos variou de acordo com a forma e a dose de administração da planta. Folhas frescas para ingestão à vontade e doses mais baixas (folhas frescas e dessecadas) levaram à evolução mais longa.

0 exame de urina realizado nos animais que adoeceram e morreram e no animal controle não revelou alterações significativas. (Quadro 4).

O animal controle (Coelho 1470) (Quadro 2) manteve-se ativo e responsivo a estímulos externos durante todo o período de experimento.

Os achados macroscópicos (Quadro 5) nos 14 coelhos que morreram consistiram em palidez e cianose acentuada de mucosas; todos os vasos encontravam-se muito dilatados e repletos de sangue, principalmente os vasos do tecido subcutâneo e a veia cava. 0 coração apresentava os vasos epicárdicos ingurgitados (todos os coelhos) e a aurícula dilatada e repleta de sangue (Coelhos 1451 e 1453) (Fig.5). 0 fígado apresentava-se pálido com evidenciação da lobulação hepática (todos os coelhos) (Fig.6). Os rins apresentavam congestão da região córtico-medular (Coelhos 1451, $1452,1453,1454,1455,1456,1457,1461,1462,1463$ e 1465).

Congestão foi o achado histopatológico (Quadro 6) mais frequente (todos os coelhos), observados em pulmão, co-

Quadro 5. Achados macroscópicos na intoxicação experimental por Metternichia princeps em coelhos

\begin{tabular}{|c|c|c|c|c|c|c|c|}
\hline \multirow{2}{*}{$\begin{array}{l}\mathrm{N}^{\circ} \text { Coelho } \\
\text { (Reg. SAP) }\end{array}$} & \multirow{2}{*}{$\frac{\text { Mucosas }}{\text { Palidez e cianose }}$} & \multirow{2}{*}{$\begin{array}{c}\text { Vasos sanguíneos } \\
\text { Dilatados e reple- } \\
\text { tos de sangue }\end{array}$} & \multicolumn{2}{|c|}{ Coração } & \multicolumn{2}{|c|}{ Fígado } & \multirow{2}{*}{$\begin{array}{c}\text { Rim } \\
\text { Região córtico- } \\
\text { medular congesta }\end{array}$} \\
\hline & & & $\begin{array}{c}\text { Vasos epicárdicos } \\
\text { ingurgitados }\end{array}$ & $\begin{array}{l}\text { Aurículas dilatadas e } \\
\text { repletas de sangue }\end{array}$ & Palidez & $\begin{array}{l}\text { Evidenciação } \\
\text { da lobulação }\end{array}$ & \\
\hline \multicolumn{8}{|c|}{ Folhas dessecadas } \\
\hline 1449 (SAP 32065) & $+{ }^{a}$ & + & + & - & + & + & - \\
\hline 1450 (SAP 32066) & + & + & + & - & $+(+)$ & ++ & - \\
\hline 1451 (SAP 32067) & ++ & + & ++ & + & ++ & ++ & + \\
\hline 1452 (SAP 32068) & + & + & + & - & ++ & ++ & + \\
\hline 1453 (SAP 32069) & + & + & ++ & + & ++ & ++ & + \\
\hline 1454 (SAP 32121) & + & + & + & - & ++ & ++ & + \\
\hline 1455 (SAP 32122) & + & + & ++ & - & ++ & ++ & \\
\hline 1456 (SAP 32071) & ++ & ++ & ++ & - & +++ & +++ & ++ \\
\hline 1457 (SAP 32072) & ++ & ++ & + & - & +++ & +++ & ++ \\
\hline \multicolumn{8}{|c|}{ Folhas maduras dessecadas e armazenadas por 6 meses } \\
\hline 1468 (SAP 32073) & +++ & ++ & ++ & - & +++ & +++ & - \\
\hline 1474 (SAP 32075) & - & - & - & - & - & - & - \\
\hline Controle & & & & & & & \\
\hline \multicolumn{8}{|c|}{ Brotos e folhas maduras frescas } \\
\hline 1461 (SAP 32073) & + & ++ & + & - & + & + & + \\
\hline 1462 (SAP 32075) & + & ++ & + & - & + & + & \\
\hline 1463 (SAP 32074) & + & ++ & + & - & + & + & \\
\hline 1465 (SAP 32124) & + & ++ & + & - & + & ++ & + \\
\hline
\end{tabular}

a +++ Lesão acentuada, ++ moderada, + leve, (+) discreta, - ausente.

Quadro 6. Achados histopatológicos observados nos experimentos realizados em coelhos com folhas de Metternichia princeps

\begin{tabular}{|c|c|c|c|c|c|c|c|c|c|c|}
\hline \multirow{2}{*}{$\begin{array}{l}\mathrm{N}^{\circ} \text { Coelho } \\
\text { (Reg. SAP) }\end{array}$} & \multicolumn{3}{|c|}{ Coração } & \multicolumn{4}{|c|}{ Fígado } & \multicolumn{3}{|c|}{ Rim } \\
\hline & Congestão & Vacuolização & $\begin{array}{l}\text { Necrose de } \\
\text { miócitos }\end{array}$ & Congestão & $\begin{array}{l}\text { Tumefação de } \\
\text { hepatócitos }\end{array}$ & Necrose & $\begin{array}{l}\text { Leucocito- } \\
\text { estase de } \\
\text { eosinófilos }\end{array}$ & Congestão & $\begin{array}{l}\text { Material } \\
\text { eosinofílico } \\
\text { dentro dos } \\
\text { túbulos }\end{array}$ & $\begin{array}{l}\text { Tumefação de } \\
\text { células epite- } \\
\text { liais dos túbu- } \\
\text { los uriníferos }\end{array}$ \\
\hline
\end{tabular}

\begin{tabular}{|c|c|c|c|c|c|c|c|c|c|c|}
\hline \multicolumn{11}{|c|}{ Folhas dessecadas } \\
\hline 1449 (SAP 32065) & $+{ }^{a}$ & + & + & $+(+)$ & + & + & $(+)$ & - & + & - \\
\hline 1450 (SAP 32066) & + & + & + & $+(+)$ & ++ & + & $(+)$ & - & ++ & - \\
\hline 1451 (SAP 32067) & $+(+)$ & + & + & ++ & +++ & + & + & $+(+)$ & + & - \\
\hline 1452 (SAP 32068) & ++ & + & ++ & ++ & +++ & + & + & + & $+(+)$ & - \\
\hline 1453 (SAP 32069) & $+(+)$ & + & $+(+)$ & ++ & +++ & + & + & $(+)$ & - & + \\
\hline 1454 (SAP 32121) & +++ & $+(+)$ & $+(+)$ & ++ & +++ & - & + & $+(+)$ & + & - \\
\hline 1455 (SAP 32122) & +++ & + & $+(+)$ & - & +++ & - & - & $(+)$ & - & - \\
\hline 1456 (SAP 32071) & ++ & + & $+(+)$ & ++ & +++ & + & + & $+(+)$ & + & - \\
\hline 1457 (SAP 32072) & +++ & $+(+)$ & $+(+)$ & ++ & +++ & - & + & ++ & - & + \\
\hline \multicolumn{11}{|c|}{ Folhas maduras dessecadas e armazenadas por 6 meses } \\
\hline 1468 (SAP 32173) & ++ & - & + & + & + & $(+)$ & - & - & - & - \\
\hline $\begin{array}{c}1474 \text { (SAP 32075) } \\
\text { Controle }\end{array}$ & - & - & - & - & - & - & - & - & - & - \\
\hline \multicolumn{11}{|c|}{ Brotos e folhas maduras frescas } \\
\hline 1461 (SAP 32073) & ++ & ++ & ++ & $+(+)$ & ++ & - & $(+)$ & + & - & + \\
\hline 1462 (SAP 32075) & ++ & $++(+)$ & ++ & $++(+)$ & ++ & - & $(+)$ & + & - & + \\
\hline 1463 (SAP 32074) & $+(+)$ & + & + & +++ & ++ & - & + & $+(+)$ & + & - \\
\hline 1465 (SAP 32124) & $+(+)$ & + & ++ & + & ++ & - & ++ & + & - & ++ \\
\hline
\end{tabular}


ração, fígado, baço, bexiga e sistema nervoso central (córtex cerebral e cerebelo). No coração, além da congestão (todos os coelhos), foi observada vacuolização de algumas fibras isoladas ou de pequenos grupos de fibras (em todos os coelhos, exceto no Coelho 1468). Também havia necrose de miócitos isolados e em grupos; as células afetadas apresentavam-se mais eosinofílicas, homogêneas e com os núcleos picnóticos ou ausentes (em todos os coelhos). No fígado de todos os animais, as lesões foram uniformes. A congestão era predominantemente centrolobular. Sinusoides, veias centrolobulares, artérias e veias porta encontravam-se dilatadas e repletas de sangue (em todos os animais exceto no Coelho 1455). 0 achado histológico mais significativo foi tumefação dos hepatócitos (em todos os coelhos) (Fig.7-8). Nos casos em que a lesão era mais grave, a distribuição era difusa, os hepatócitos apresentavam-se aumentados de volume, com citoplasma rarefeito e claro ou levemente granular e até com aspecto espumoso, e os núcleos vesiculosos e com a cromatina marginada. Em alguns casos, os hepatócitos apresentavam-se tumefeitos somente na região centrolobular (Coelhos 1449 e 1450). Havia necrose incipiente focal de hepatócitos ou necrose

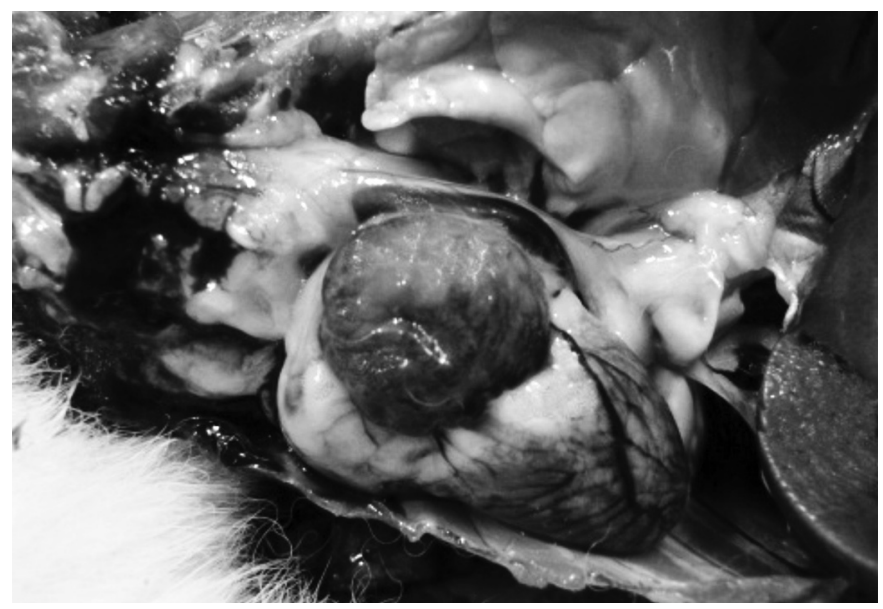

Fig.5. Aurícula e vasos do coração dilatados e repletos de sangue na intoxicação experimental $(0,25 \mathrm{~g} / \mathrm{kg})$ por Metternichia princeps no Coelho 1453 (SAP 32069).

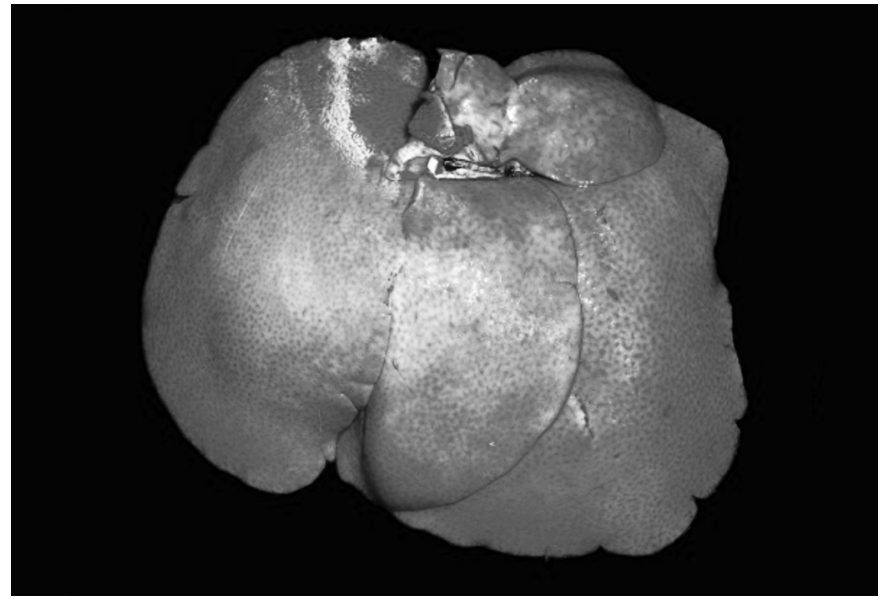

Fig.6. Fígado difusamente pálido e com evidenciação da lobulação na intoxicação experimental $(1 \mathrm{~g} / \mathrm{kg})$ por Metternichia princeps no Coelho 1468 (SAP 32173).

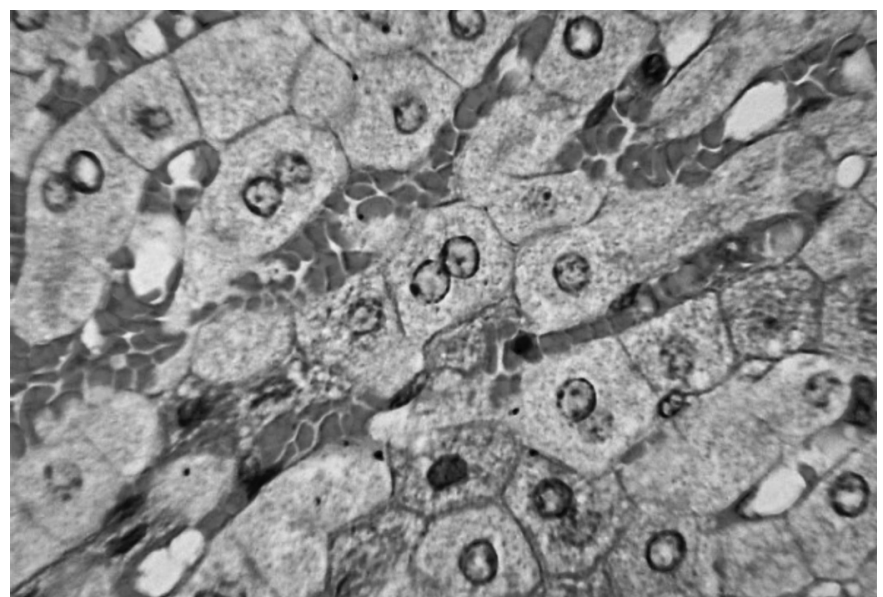

Fig.7. Congestão e tumefação difusa de hepatócitos na intoxicação experimental $(2,4 \mathrm{~g} / \mathrm{kg})$ por Metternichia princeps no Coelho 1457 (SAP 32074). HE, obj.40x.

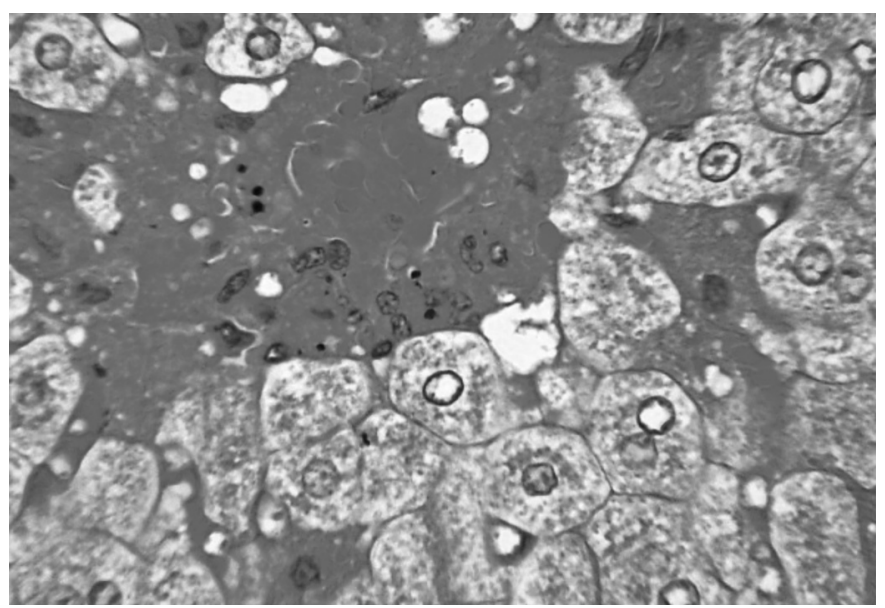

Fig.8. Congestão, tumefação e necrose hepática na região portal na intoxicação experimental $(0,25 \mathrm{~g} / \mathrm{kg})$ por Metternichia princeps no Coelho 1453 (SAP 32069). HE, obj.40x.

de pequenos grupos de hepatócitos ou de hepatócitos isolados que se encontravam com citoplasma eosinofílico e núcleo picnótico, com maior frequência na região centrolobular, mas também era observada na região peri-portal (Coelhos 1449, 1450, 1451, 1452, 1453, 1456 e 1468) (Fig.8). A coloração pelo ácido periódico de Schiff (PAS) do fígado dos Coelhos 1469 e 1470 resultou negativa. Leucocitoestase de eosinófilos foi constatada (em todos os casos, exceto no Coelho 1455). No rim, foi observada, além da congestão (em todos os coelhos exceto Coelhos 1449, 1450 e 1468), proteína na luz dos túbulos renais em parte dos animais (Coelhos 1449, 1450, 1451, 1452, 1454, 1456, 1463). Em alguns casos, também ocorreu tumefação das células tubulares renais., com citoplasma granular, sem, contudo, evidenciarem alterações nucleares (Coelhos $1453,1457,1461,1462,1465)$.

\section{DISCUSSÃO}

Foi verificado que o coelho é sensível à intoxicação por Metternichia princeps; no quadro clínico-patológico predominavam alterações relativas ao coração, ao fígado e, com menor intensidade, aos rins. 
Dois coelhos (Coelhos 1454 e 1455) que adoeceram mas se recuperaram, dois meses mais tarde receberam novamente a planta e morreram, o que indica que não há desenvolvimento de tolerância à toxidez da planta (Quadro 1).

Dos seis coelhos que receberam brotos frescos, três que ingeriram respectivamente $1,55 \mathrm{~g} / \mathrm{kg}, 2,4 \mathrm{~g} / \mathrm{kg}$ e $3 \mathrm{~g} / \mathrm{kg}$, morreram, enquanto outros três coelhos, que ingeriram $1,8 \mathrm{~g} / \mathrm{kg}$, $2,36 \mathrm{~g} / \mathrm{kg}$ e $2,8 \mathrm{~g} / \mathrm{kg}$, não morreram.

Dos quatro coelhos que receberam as folhas maduras frescas, só morreu um coelho (Coelho 1465), o que ingeriu $2 \mathrm{~g} / \mathrm{kg}$, os outros três (Coelhos 1464, 1466 e 1467) que ingeriram respectivamente $1,78 \mathrm{~g} / \mathrm{kg}, 1,24 \mathrm{~g} / \mathrm{kg}$ e $1,22 \mathrm{~g} / \mathrm{kg}$, não adoeceram. Uma comparação entre a toxidez dos brotos frescos (doses letais de $1,55 \mathrm{~g} / \mathrm{kg}, 2,4 \mathrm{~g} / \mathrm{kg}$ e $3 \mathrm{~g} / \mathrm{kg}$ e não letais de $2,36 \mathrm{~g} / \mathrm{kg}, 1,8 \mathrm{~g} / \mathrm{kg}$ e $2,8 \mathrm{~g} / \mathrm{kg}$ ) e das folhas maduras frescas (dose letal de $2 \mathrm{~g} / \mathrm{kg}$ e doses não letais de $1,78 \mathrm{~g} / \mathrm{kg}$, $1,24 \mathrm{~g} / \mathrm{kg}$ e $1,22 \mathrm{~g} / \mathrm{kg}$ ), indica que não há diferença entre eles. (Quadro 3)

Numa comparação entre as folhas maduras dessecadas e as folhas maduras frescas, levando em consideração que a dose da planta dessecada tem que ser multiplicada por três, as folhas maduras dessecadas causaram a morte dos coelhos em doses menores do que as folhas maduras frescas. Para as folhas dessecadas, estas foram doses correspondentes a $0,375 \mathrm{~g} / \mathrm{kg}$ a $0,75 \mathrm{~g} / \mathrm{kg}$ da planta fresca e para as folhas frescas (brotos e folhas maduras) foram doses a partir de 1,55g/kg. Uma possível explicação para esta discrepância, poderia ser o fato que a planta dessecada foi administrada sob forma de pó em período de minutos e desta forma era mais rapidamente absorvida pela mucosa do trato digestório do que a folha fresca, que era ingerida durante um a dois dias.

O quadro clínico observado nos coelhos intoxicados por M. princeps foi uniforme entre todos os animais, tanto nos que receberam a planta dessecada quanto nos que ingeriram folhas verdes maduras e brotos. A análise da urina dos coelhos que adoeceram não revelou alterações; todos os parâmetros avaliados foram semelhantes ao animal controle. Nos caprinos intoxicados naturalmente por M. princeps, a avaliação sorológica indicou lesão renal através do aumento dos níveis de ureia e creatinina, o que confirma a ação nefrotóxica da planta nos caprinos (Prado 2012).

A evolução da intoxicação por $M$. princeps em coelhos caracterizou-se por morte superaguda a aguda e mostrou-se mais longa ao ser comparada com a insuficiência cardíaca causada pela intoxicação por plantas que causam morte súbita. Nestas o quadro observado caracteriza-se pelo súbito aparecimento dos sinais clínicos, quando animais aparentemente sadios começam a debater-se, fazem movimentos bruscos, violentos e desordenados, caem em decúbito lateral e logo morrem, como foi relatado nas intoxicações experimentais em coelhos por Palicourea marcgravii (Pacheco \& Carneiro 1932, Peixoto et al. 1987), P. juruana (Tokarnia \& Döbereiner 1982), P. grandiflora, (Döbereiner \& Tokarnia 1982), Pseudocalymma elegans (Tavares et al. 1974), Arrabidea japurensis (Döbereiner \& Tokarnia 1983), A. bilabiata (Döbereiner et al. 1984), Mascagnia pubiflora (Döbereiner et al. 1986), Mascagnia aff. rigida (Tokarnia et al. 1985), M. rigida (Tokarnia et al. 1987) e M. exotropica (Tokarnia et al. 1998).
No fígado, a lesão histológica mais relevante na intoxicação por M. princeps consiste em tumefação dos hepatócitos. No coração, a lesão histológica mais evidente, observada em todos os coelhos, foi congestão. A vacuolização e eosinofilia de fibras miocárdicas, embora também tenha ocorrido em todos os coelhos era incipiente e em pequenos grupos de miócitos. Essa alteração tem sido descrita em casos de insuficiência cardíaca aguda em ruminantes (Newsholme \& Coetzer 1984). Stigger et al. (2001) também observaram esta alteração em corações de ovinos normais e sugerem que é provável que se trate de artefatos causados pela contração da fibra no fixador. Ainda esses autores, citando Robinson \& Maxie (1993), afirmam que a eosinofilia dos cardiomiócitos também é uma alteração precoce causada pela isquemia. Em nossos experimentos com M. princeps, todos os coelhos apresentaram sinais clínicos compatíveis com insuficiência cardíaca aguda, mas a possibilidade de que as lesões nas fibras miocárdicas estejam associadas a artefatos não pode ser excluída.

Além da degeneração albuminosa-granular de células epiteliais tubulares renais, observou-se na intoxicação por Thiloa glaucocarpa necrose das células epiteliais de alguns poucos túbulos isolados, em dois de nove coelhos (Tokarnia et al. 1988), o que não foi observado nos coelhos intoxicados por M. princeps.

As alterações hepáticas e cardíacas sugerem que a morte dos animais esteja intimamente relacionada com a falha do coração. Presume-se que a ação direta do princípio tóxico possa acarretar insuficiência cardíaca aguda e consequente choque cardiogênico.

Acredita-se que a ação indireta da planta resulte nas alterações histopatológicas hepáticas e renais, decorrentes de lesão celular provocada pela hipóxia. A vacuolização dos hepatócitos e necrose de células individuais e em grupos observados nos nossos experimentos também aparecem em animais intoxicados por T. glaucocarpa e pelas plantas do grupo das que causam morte súbita.

\section{REFERÊNCIAS}

Caldas S.A. 2012. Intoxicação experimental por Meternichia princeps (Solanaceae) em bovinos e ovinos. (Comunicação pessoal, Médico Veterinário, Barra do Piraí, RJ)

Caldas S.A., Prado J.S., Costa S.Z.R., Peixoto T.C., Brito M.F. \& Tokarnia C.H. 2011. Intoxicação natural por Metternichia princeps (Solanaceae) em caprinos. XV Enapave, Goiânia, GO. (Resumo)

Döbereiner J. \& Tokarnia C.H. 1982. Intoxicação experimental por Palicourea grandiflora (Rubiaceae) em coelhos. Pesq. Vet. Bras. 2(3):121-124.

Döbereiner J. \& Tokarnia C.H. 1983. Intoxicação experimental por Arrabidaea japurensis (Bignoniaceae) em coelhos. Pesq. Vet. Bras. 3(3):95-97.

Döbereiner J., Peixoto P.V. \& Tokarnia C.H. 1984. Intoxicação experimental por Arrabiadaea bilabiata em coelhos. Pesq. Vet. Bras. 4(3):89-96.

Döbereiner J., Gava A., Consorte L.B. \& Tokarnia C.H. 1986. Intoxicação experimental por Mascagnia pubiflora (Malpighiaceae) em coelhos. Pesq. Vet. Bras. 6(2):51-57.

Newsholme S.J. \& Coetzer J.A.W. 1984. Myocardial pathology of domestic ruminants in Southern Africa. J. South Africa Vet. Med. Assoc. 55:89-96.

Pacheco G. \& Carneiro V. 1932. Estudos experimentais sobre plantas tóxicas. I. Intoxicação dos animais pela "erva de rato da mata". Revta Soc. Paulista Med. Vet. 2(2/3):23-46.

Peixoto P.V., Tokarnia C.H., Döbereiner J. \& Peixoto C.S. 1987. Intoxicação 
experimental por Palicourea marcgravii (Rubiaceae) em coelhos. Pesq. Vet. Bras. 7(4):117-129.

Peixoto P.V., Brust L.A.C., Brito M.F., França T.N., Cunha B.R.M. \& Andrade G. B. 2003. Intoxicação natural por Amaranthus spinosus (Amaranthaceae) em ovinos no Sudeste do Brasil. Pesq. Vet. Bras. 23(4):179-184.

Prado J.S. 2012. Intoxicação natural e experimental por Metternichia princeps (Solanaceae) em caprinos. Dissertação de Mestrado, UFRRJ, Rio de Janeiro, RJ.

Robinson W.F. \& Maxie M.G. 1993.The cardiovascular system, p.1-100. In: Jubb K.V.F., Kennedy P.C. \& Palmer N. (Eds), Pathology of Domestic Animals. Vol.3. $4^{\text {th }}$ ed. Academic Press, San Diego. 653p.

Stigger A.L., Barros C.S.L., Langohr I.M. \& Barros S.S. 2001. Intoxicação experimental por Ateleia glazioviana (Leg. Papilionoideae) em ovinos. Pesq. Vet. Bras. 21(3):98-108.

Tavares M.I., Rezende A.M.L. \& Döbereiner J. 1974. Intoxicação experimental por Pseudocalymma elegans em coelhos e cobaias. Pesq. Agropec. Bras., Sér. Vet. 9:91-94.
Tokarnia C.H. \& Döbereiner J. 1982. Intoxicação experimental por Palicourea juruana (Rubiaceae) em bovinos e coelhos. Pesq. Vet. Bras. 2(1):1726.

Tokarnia C.H., Peixoto P.V. \& Döbereiner J. 1985. Intoxicação experimental por Mascagnia aff. rigida (Malpighiaceae) em coelhos. Pesq. Vet. Bras. 5(4):121-128.

Tokarnia C.H., Döbereiner J. \& Canella C.F.C. 1987. Intoxicação por Mascagnia rigida (Malpighiaceae) em coelhos. Pesq. Vet. Bras. 7(1):11-16.

Tokarnia C.H., Peixoto P.V. \& Döbereiner J. 1988. Intoxicação experimental pelas folhas e extratos de Thiloa glaucocarpa (Combretaceae) em coelhos. Pesq. Vet. Bras. 8(3/4):61-74.

Tokarnia C.H., Gava A., Brito M.F., Bezerra P.S. \& Oliveira K.D. 1998. Intoxicação experimental em coelhos por Mascagnia sp. (Malpighiaceae) procedente do Estado de Santa Catarina. Pesq. Vet. Bras. 18(2):61-64.

Tokarnia C.H., Brito M.F., Barbosa J.D., Peixoto P.V. \& Döbereiner J. 2012. Plantas Tóxicas do Brasil para Animais de Produção. $2^{\text {a }}$ ed., Editora Helianthus, Rio de Janeiro. 586p. 